\title{
Slow potassium dynamics and seizure evolution
}

\section{Rob Cressman Jr*1, Ghanim Ullah ${ }^{1}$, Jokubas Ziburkus ${ }^{2}$, Ernest Barreto ${ }^{3}$ and Steven J Schiff 1,3}

\author{
Address: ${ }^{1}$ Center for Neural Engineering, Department of Engineering Science and Mechanics, Pennsylvania State University, University Park, PA \\ 16802, USA, ${ }^{2}$ University of Houston, Houston, TX 77004, USA and ${ }^{3}$ Krasnow Institute for Advanced Study, George Mason University, Fairfax, VA \\ 22030, USA and Department of Neurosurgery, Pennsylvania State University, University Park, PA 16802, USA \\ Email: Rob Cressman* - jrc22@psu.edu \\ * Corresponding author
}

from Sixteenth Annual Computational Neuroscience Meeting: CNS*2007

Toronto, Canada. 7-12 July 2007

Published: 6 July 2007

BMC Neuroscience 2007, 8(Suppl 2):P80 doi:I0.I I86/I47|-2202-8-S2-P80
(C) 2007 Cressman et al; licensee BioMed Central Ltd.

Seizures involve dynamics on a wide range of temporal scales, from spike times on the order of milliseconds to the large depolarizations seen in single cells that can last several tens of seconds. At the longest time scales, these events modify the cellular environment, altering oxygen, potassium, sodium and other electrolyte concentrations to produce a durable but transient modification of the network dynamics. In order to investigate these slow dynamics we have developed a highly simplified model that monitors the changes in ionic concentrations in and around highly active cells, while disregarding the fast dynamics responsible for action potential generation. We model the time-dependent potassium concentration in and around a cell resulting from flow through voltagegated channels, pumps, and the surrounding glial network. The flow through voltage-gated channels is determined by time-averaging simulated potassium currents in a Hodgkin-Huxley conductance-based neuron. The current is a function of both intra- and extracellular potassium concentrations and responds to changes in the concentration gradient over a duration that is long compared to the time associated with spiking events. On the other hand, this response time, which can be as slow as a fraction of a second, is still short compared to the lifetime of a network seizure and can be considered instantaneous. Therefore we disregard the response time and approximate the model as a pair of differential equations which are amenable to a complete phase plane analysis. We report on the results of this phase plane analysis and show comparisons with results from in vitro experiments. 